\title{
CONDUITE INTENSIVE DES TROUPEAUX OVINS. EFFETS DU TARISSEMENT DÈS LA MISE BAS SUR LA FÉCONDITÉ DE BREBIS INSÉMINÉES TOUS LES 6 MOIS
}

\author{
M. THÉRIEZ et G. MOLÉNAT \\ avec la collaboration de \\ A. Brelurut, P. Dacheux, J.-M. Mansart et M. Maguère \\ Station de Recherches sur l'Élevage des Ruminants, \\ Centre de Recherches de Clermont-Ferrand, I. N.R. A., \\ Theix, 63110 Beaumont
}

\section{RÉSUMÉ}

\begin{abstract}
Pendant 4 années successives nous avons soumis un troupeau de brebis Charmoises et croisées Romanov $\times$ Limousines à un plan de conduite intensif. Les brebis étaient inséminées $5^{\circ}$ jours après mise bas après traitement progestatif et injection de PMSG. Nous avons comparé la fertilité, la prolificité et la fécondité des brebis qui allaitaient leurs agneaux ou qui avaient été taries dès la mise bas. Ces dernières sont en moyenne plus fertiles ( $8 \mathrm{p}$. Ioo, différence NS) que les brebis allaitantes, mais l'importance de la différence varie selon la race et la saison de reproduction. Les brebis inséminées 50 jours après mise bas sont moins fertiles et moins prolifiques que celles qui ont agnelé plus de 6 mois avant l'insémination artificielle. Les brebis Charmoises ont produit en moyenne I,80 agneau par femelle et par an avec I,3 agnelage par an et les croisées 2,70 agneaux avec I,2I agnelage femelle/an.
\end{abstract}

\section{IN'TRODUC'TION}

L'utilisation d'éponges vaginales imprégnées de progestatifs permet de féconder des brebis en période d'anœestrus post partum ou saisonnier (Robinson, I965 ; ThimoNIER et al., I968) et, par là, de réduire l'intervalle entre deux mises bas successives. Thimonier et MAUı Éon (I969) ont proposé un plan de conduite du troupeau basé sur l'emploi de cette technique avec des agnelages tous les igo jours. Mis en service sur un troupeau de brebis Ile de France et Préalpes, ce plan de conduite leur a permis 
d'obtenir respectivement 0,32 et 0,52 agnelage supplémentaire par brebis et par an, soit environ 0,6 et I agneau de plus par brebis/an par rapport à un système avec agnelage tous les 12 mois.

Cependant, la fertilité ( ${ }^{1}$ ) des brebis ainsi inséminées en anœstrus post partum est faible ; elle serait inférieure de $\mathrm{I} 4 \mathrm{p}$. Ioo environ à celle des brebis sèches, l'écart étant plus important au printemps qu'à l'automne (THIMONIÉ et al., I968). Cette dernière observation est en accord avec celles de LAMming (I96I) et de RaHman et KitTS (I967), elle a été confirmée par Cognie, Cornu et Maulíon (I974).

Pour améliorer le taux de fertilité du troupeau on peut envisager de tarir les brebis avant le début du traitement progestatif, c'est-à-dire après 30 à 35 jours de lactation. Le tarissement nécessite alors une réduction importante des apports alimentaires à une période où il serait, au contraire, souhaitable de les augmenter pour améliorer la fertilité et la prolificité des animaux (flushing). Car, même avec l'emploi d'éponges vaginaies et de PMS, le flushing est une technique d'élevage intéressante qui permet d'améliorer d'environ 0,2 le taux de fécondité (Girou et al., I97I).

On peut également tarir les brebis dès la mise bas et élever les agneaux artificiellement.

L'expérience entreprise avait pour objectif de déterminer les effets du sevrage précoce ( $\mathrm{I} 2$ à $24 \mathrm{~h}$ après mise bas) sur la fécondité d'un troupeau constitué de brebis de deux races différentes (Charmoises à faible prolificité et croisées Romanov $\times$ Limousines à prolificité élevée) conduit selon un rythme de reproduction intensif (deux agnelages par an), avec un emploi de progestatifs de synthèse. Les animaux affectés à l'un des deux lots expérimentaux (sevrage précoce $24 \mathrm{~h}$ après mise bas ou allaitement) lors de leur premier agnelage, ont subi le même traitement tout au long de l'expérience. Celle-ci a débuté au printemps rg6g et s'est terminée durant l'été I973.

\title{
TABLEAU I
}

\section{Définition des termes utilisés}

\author{
Taux de fertilité : nombre de brebis agnelant/nombre de brebis inséminées $\times 100$ \\ Fertility rate: number of lambing ewes/number of ewes inseminated $\times 100$ \\ Taux de prolificité : nombre d'agneaux nós/nombre de brebis agnelant $\times 100$ \\ Prolificacy rate: number of lambs born/number of ewes lambing $\times 100$ \\ Taux de fécondité : nombre d'agneaux nés/nombre de brebis insémincées $\times 100$ \\ Fecundity rate: number of lambs born/mumber of ewes inseminated $\times 100$ \\ Brebis sc̀che : brebis n'ayant pas agnelé \\ Dry exe : ewe which failed to lamb \\ Brebis mère : brebis ayant agnele 45 jours avant IA (qu'clle allaite ou non) \\ Post partum ewe : ewe which lambed 45 days before $A I$ (wether lactating or not) \\ Jrebis tarie: brebis mère tarie dès la mise bas \\ Severed ewe: etee which lambed and whose lamb has been weaned 24 hours after birth \\ Brebis allaitante : brebis mère allaitant son ou ses agneaux \\ Lactating ewe : ewe nursing her lambs
}

Agnelle : femelle à son premier agnelage, quel que soit son âge

First lamb etee : fomale at her first lambing.

(1) L'ensemble des termes utilisés sit défuni dans lo tableatu 1. 


\section{MATÉRIEI, ETT MÉTHODES}

Nous avons comparé, au cours de 5 années successives, les taux de fertilité, de prolificité et de fécondité de deux lots d'animaux, composés chacun de brebis Charmoises et croisées Romanov $\times$ Limousines qui allaitaient leurs agneaux pendant 8 semaines (lot témoin) ou étaient taries dès la mise bas (lot expérimental). Les animaux recevaient le même régime alimentaire en dehors de la période de lactation. Los résultats se rapportent à toutes les brebis vivantes lors des inséminations et à tous les ağneaux conçus.

\section{Plan de conduite du troupeau}

Nous avons soumis l'ensemble des brebis au plan de conduite intensive proposé par ThImoNIER et Mauléon (1969) avec deux périodes de lutte au cours de l'année : l'une en saison sexuelle, en octobre et novembre (lutte d'automne), l'autre en période d'anoestrus saisonnier, en avril et mai (lutte de printemps).

Lors de chaque période de lutte, les brebis sont inséminées après synchronisation des oestrus puis mises en présence de béliers $I_{4}$ jours plus tard, selon le schéma reporté sur la figure $I_{\text {. Le plan }}$ de conduite prévoit 7 périodes d'insémination par an $: 4$ à l'automne, 3 au printemps.

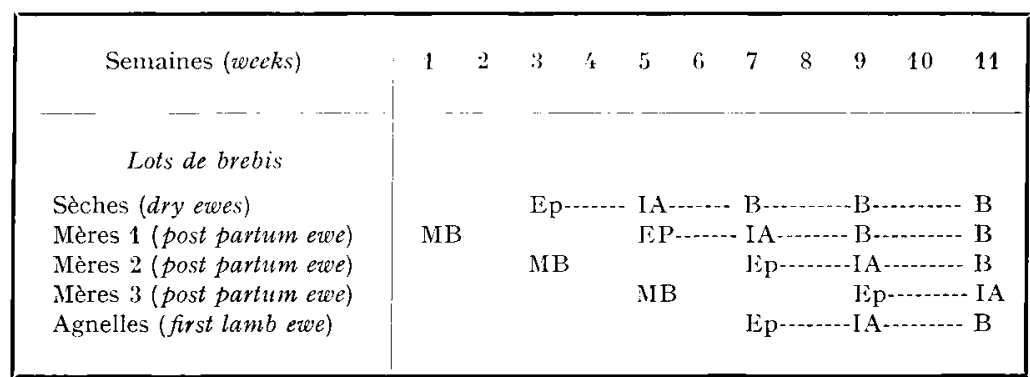

FIG. I. - Plan de conduite des luttes d'automne ${ }^{\left({ }^{1}\right)}$

(Breeding management of the flock during autumn)

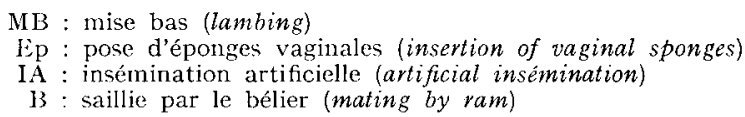

(1) Lors des luttes de printemps trois moments de lutte sont prévus. Les brebis sèches sont alors inséminées avec le premier lot de brebis mères.

(During the spring breeding period, three dates of mating were planned. Dry ewes were therefore inseminated with the first group of post partum ewes).

\section{Traitements hormonaux et insémination}

Toutes les brebis subissent le mème traitement, quel que soit leur état physiologique : sèches, taries ou allaitantes. Les éponges vaginales imprégnées de $30 \mathrm{mg}$ de liGA (acétate de fluorogestone) pour les brebis, 4o mg pour les agnelles, sont laissées en place pendant i jours. Lors du retrait, les animaux (brebis et agnelles), reçoivent une injection de PMSG (400 UI à l'automne, 600 au printemps), identique pour les animaux des deux génotypes, parce que nous ne disposions pas, en r 969 , de données permettant de la moduler selon la sensibilité de la race. L'insémination a lieu à l'aide de sperme frais, $4^{8}$ et $5^{6} \mathrm{~h}$ après le retrait des éponges, sans détection préalable des chaleurs.

Après examen dans le tube de récolte, les éjaculats retenus, sans détermination de la concentration, sont dilués à raison de I volume de sperme pour 4 volumes d'un dilueur préparé selon la méthode de Colas (I969). Les brebis reçoivent, lors cle chaque insémination $0,5 \mathrm{ml}$ du sperme 
dilué. Au cours d’une même journée d'insémination, les éjaculats provenant de chaque bélier sont répartis entre les lots de femelles (taries, allaitantes et agnelles) pour éliminer l'influence du mâle sur la fertilité.

\section{Animaux}

L'expérience a débuté en avril 1969 avec l'insémination de 35 agnelles de race Charmoise, âgées de I an. Au cours des périodes de lutte successives, nous avons introduit de nouvelles agnelles de race Charmoise, âgées également de $\mathrm{I}$ an, sans tenir compte de leur poids, et des agnelles croisées Romanov $\times$ Limousines. Celles-ci pesaient $33 \mathrm{~kg}$ au minimum lors de leur première lutte, elles étaient âgées de 7 mois pour les plus précoces ( 84 animaux), de Io mois au plus pour les autres (I05 animaux). Les effectifs utilisés pour l'expérience sont reportés sur le tableau 2.

\section{TABLEAU 2}

Nombre d'animaux mis en expérience

Number of animals used in experiment

\begin{tabular}{|c|c|c|c|c|c|c|c|c|}
\hline \multicolumn{2}{|c|}{$\begin{array}{l}\text { Année de naissance } \\
\text { (Year of birth) }\end{array}$} & 1967 & 1968 & 1969 & 1970 & $1 \$ 71$ & Total & $\begin{array}{l}\text { Poids moyen } \\
\text { ̀̀ la } 1^{\text {re lutte }} \\
\text { (Mean weight }\end{array}$ \\
\hline $\begin{array}{c}\text { Nombre } \\
\text { d'animaux } \\
\text { (Number } \\
\text { of animals) }\end{array}$ & $\begin{array}{c}\text { Charmoises } \\
\text { Croisées } \\
\text { Romanov } \times \\
\text { Limousines }\end{array}$ & 12 & 34 & 23 & 104 & 20) & 112 & $\begin{array}{l}35,2 \mathrm{~kg} \text { (12 mois) } \\
33,8 \mathrm{~kg} \text { (7 mois) }\end{array}$ \\
\hline
\end{tabular}

Alimentation et conduite des animaux

Rentrées en bergerie au cours de la dernière semaine de septembre, les brebis disposaient, pendant toute la période de stabulation, de foin de pré de qualité moyenne offert à volonté et, selon leur stade physiologique, d'une quantité variable d'un aliment concentré composé de 80 p. Ioo de céréales, de i $5 \mathrm{p}$. Ioo de tourteaux, et de minéraux.

Cet aliment était offert selon le plan de rationnement suivant : $300 \mathrm{~g} / \mathrm{j}$ au cours du dernier mois de gestation, $600 \mathrm{~g} / \mathrm{j}$ pendant les 8 premières semaines de lactation. Pendant les 3 semaines précédant et les 3 semaines suivant l'insémination, les brebis taries ou sèches recevaient $300 \mathrm{~g} / \mathrm{j}$ de cet aliment concentré.

Les animaux étaient remis à l'herbe au printemps, à une date variable comprise entre le $\mathbf{I}^{\text {er }}$ avril et le I $^{\mathrm{er}}$ mai selon les conditions de l'année, mais toujours après tarissement pour les brebis allaitantes et au moins une semaine après l'insémination pour les autres. En été, les 2 lots étaient conduits ensemble sur des prairies naturelles et ne recevaient aucun complément.

Le tarissement des mères avait lieu brutalement par séparation de l'agneau I 2 à $24 \mathrm{~h}$ après la mise bas pour les brebis taries ; il était au contraire progressif au cours de la $7^{\mathrm{e}}$ semaine de lactation pour les brebis allaitantes.

\section{RÉSULTATS}

Nous avons utilisé 3 or brebis qui ont été introduites progressivement dans le troupeau. Au printemps I973, à l'issue de l'expérience, les animaux étaient âgés de I an et demi à 6 ans et demi pour les Charmoises et de $\mathbf{I}$ an et demi à 4 ans et demi pour les croisées Romanov $\times$ Limousines. 
l.e nombre d'inséminations par femelle a varjé de 2 à $i$ pour les premières et de 2 à 7 pour les secondes. L'intervalle moyen entre la mise bas et la prenière insémination a été au printemps de $47,3 \mathrm{j}( \pm 6,7)$ pour les brebis allaitantes, de $48,5 \mathrm{j}( \pm 7,5)$ pour les taries et, respectivement, de $49,0 \mathrm{j}( \pm 6,0)$ et $50,3 \mathrm{j}( \pm 8,1)$ pour ces mêmes brebis à l'automne.

\section{Effrts du tarissement sur le taux de réforme et le poids des brebis}

Les brebis utilisées au cours de cette expérience étant jeunes, nous n'avons pas eu de réformes pour mauvais état général ; les seules causes d'élimination ont été les pertes ou accidents $(9 \mathrm{croi}-$ sées Romanov $\times$ limousines et 22 Chamoises).

De même il n'a pas été possible de mettre en évidence une fatigue des animaux conduits selon le ry thme intensif. Lors de l'agnelage de printemps 1972, nous avons pesé toutes les brebis 24 heures après la mise bas. Les animaux qui avaient allaité leurs agneaux à l'issue des gestations antérieures étaient systématiquement plus lourds que ceux qui n'avaient effectué aucune lactation (tabl. 3). L'écart est significatif $(\mathrm{P}<0,05)$ chez les Romanov $\times$ Limousines de 2 ans et chez les Chavmoises de 3 ans. Il est hautement significatif $(\mathrm{P}<0, \mathrm{OI})$ lorsque l'on compare l'ensemble des animaux de chaque catégorie.

\section{TABLEAU 3}

Poids moyen des brebis 24 heures après l'agnelage (printemps 1972) selon leur mode de conduite

Mean weight of the ewes 24 hours after lambing (spring 1972) according to experimental treatment

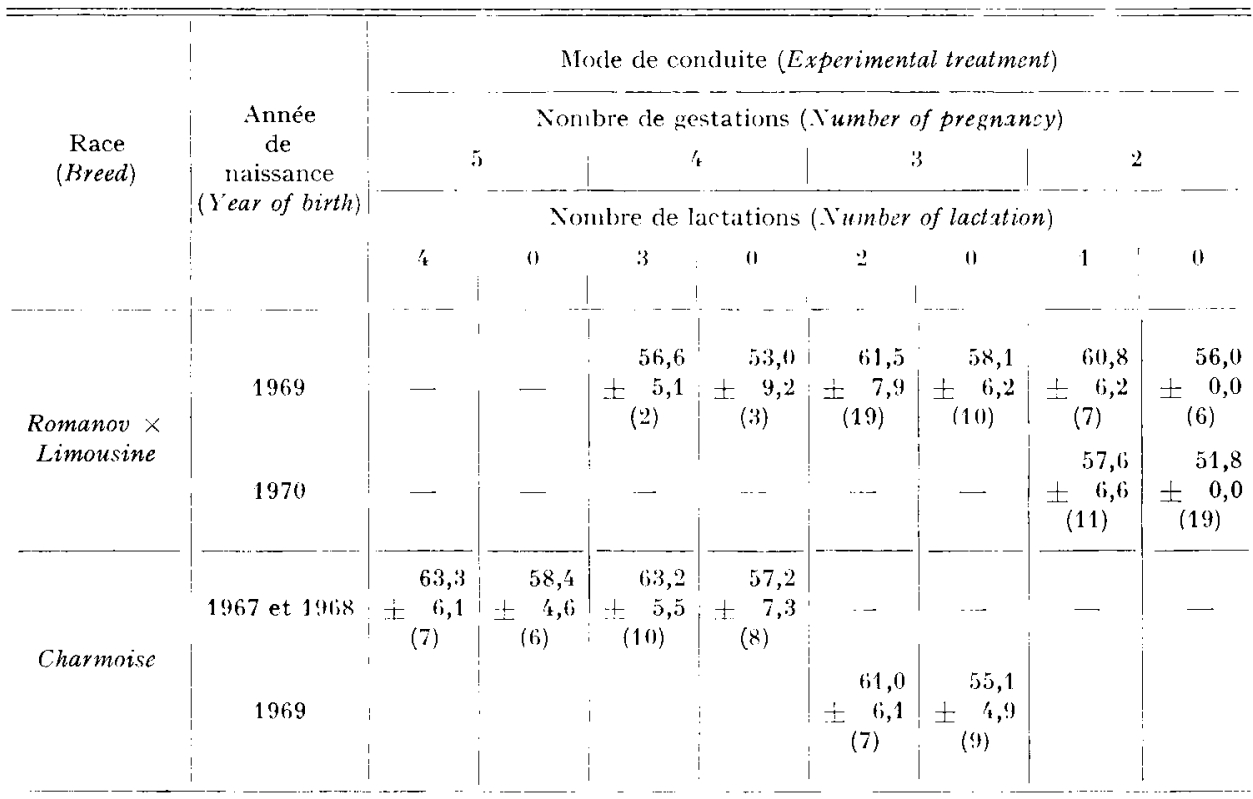

( ) Nombre d'animaux (Number of animals).

Effets du tarissement sur la fertilité, la prolificité et la fécondité des brebis

Au printemps, le tarissement dès la mise bas a amélioré significativement $(\mathrm{P}<0,05)$ la fertilité après IA des brebis Romanov $\times$ Limousines $(60,2$ contre 43,8 p. 1oo) ; il n'a pas eu d'effet sur les brebis Charmoises. 
M. THÉRIEZ, G. MOLÉNAT

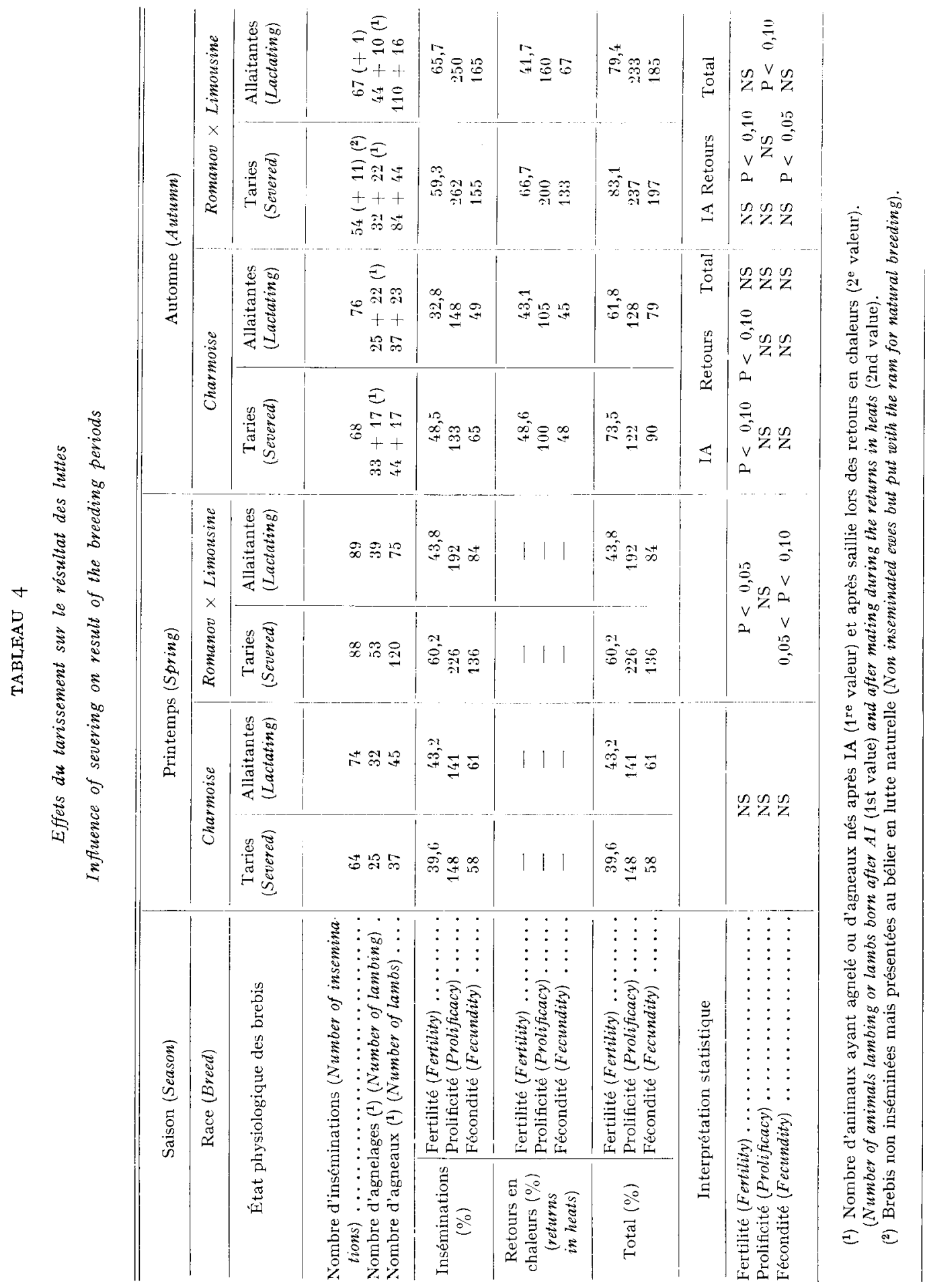


En automne, la fertilité après IA des brebis Charmoises taries est supérieure à celle des brebis allaitantes $(48,5$ contre $32,8 \mathrm{p}$. IOO, différence au seuil de signification $0,05<\mathrm{P}<0, \mathrm{IO})$. Par contre elle est plus faible chez les brebis croisées Romanov $\times$ Limousines taries que chez les allaitantes $(59,3$ contre 65,7 p. Ioo - différence non significative). Dans les deux races les brebis taries sont plus fertiles que les allaitantes après saillie lors des retours en chaleurs, mais les différences sont au seuil de la signification. Sur l'ensemble IA et saillies lors des retours en chaleurs, nous avons observé la même tendance dans chaque race, mais les différences ne sont pas significatives quelle que soit la race.

Si l'on regroupe les données obtenues avec les deux génotypes, la fertilité après IA a été, respectivement pour les brebis allaitantes et taries, 43,5 et $5 \mathrm{I}, 3 \mathrm{p}$. roo au printemps, 48,3 et $53,3 \mathrm{p}$. Ioo en automne soit 45,8 et 52,2 p. Ioo pour l'ensemble de l'année. Aucune de ces différences n'est significative. La fertilité, après saillie lors des retours en chaleurs, est significativement plus élevée chez les brebis taries que chez les allaitantes : 78,2 et 70,3 p. I00 $(\mathrm{P}<0,05)$.

Toutes saisons confondues et pour l'ensemble des deux races, la fertilité des brebis taries $(63,9$ p. Ioo) est supérieure à celle des brebis allaitantes $(56,0$ p. Ioo) mais la différence est à la limite du seuil de signification $(0,05<\mathrm{P}<0,10)$.

Le tarissement n'a pas eu d'effet significatif sur la prolificité des brebis. La fécondité des brebis croisées Romanov $\times$ Limousines taries est supérieure à celle des brebis allaitantes après IA au printemps, et après saillie lors des retours en chaleurs en automne. Dans ces deux cas, ceci correspond à une meilleure fertilité des brebis. Nous n'avons pas observé de différence de fécondité entre brebis taries ou allaitantes dans le troupeau de Charmoises.

\section{Effets de l'intevvalle mise bas-insémination sur la fertilité, la prolificité et la fécondité}

Les brebis sèches sont plus fertiles que les brebis mères. La fertilité après IA est significativement plus élevée au printemps pour les Charmoises (73,9 contre 4I,3 p. Ioo) et en automne pour les deux races (respectivement 72,2 et 40,2 p. roo pour les Charmoises; 79,9 et $6 \mathrm{r}, \mathrm{I}$ p. Ioo pour les croisées Romanov $\times$ Limousines $)$.

La fertilité après IA des croisées Romanov $\times$ Limousine au printemps est très voisine dans les lots de brebis sèches et de brebis mères (53,8 et 51,9 p. Ioo). Cependant, dans ce cas, le nombre de brebis sèches inséminées ( 13 animaux) est très faible et if est difficile de tirer une conclusion de ces résultats.

Lors des saillies sur retours en chaleurs, les brebis sèches sont plus fertiles que les brebis mères, mais la différence est non significative (Charmoises) ou à la limite de la signification (croisées Romanov $\times$ Limousines).

La prolificité des brebis sèches est plus élevée que celle des brebis mères, les différences sont cependant limitées et rarement significatives, sauf dans le cas des brebis Charmoises en lutte de printemps. Les brebis sèches ont alors un taux de prolificité supérieur à 2, valeur très élevée pour la race. Ceci pourrait correspondre à un excès de la dose de PMS plutôt qu'à un effet propre du stade physiologique des femelles.

La fécondité des brebis sèches est supérieure à celle des brebis mères, tant après IA qu'après saillie, tant au printemps qu'en automne. Seule exception, les brebis croisées Romanov $\times$ Limousines au printemps, mais cela correspond aux faibles différences de fertilité observées entre les lots de brebis sèches et brebis mères de cette race.

\section{Effets du nombre d'agneaux allaités}

Le nombre d'agneaux allaités n'a pas eu d'effet significatif sur la fertilité, la prolificité et la fécondité des brebis des deux races lors des inséminations de printemps (tabl. 6). Lors des luttes d'automne, il n'en a pas eu sur la fertilité des brebis mais les bessonnières étaient moins prolifiques ( 2 I 7 contre $27 \mathrm{r}$ p. Ioo pour les croisées Romanov $\times$ Limousines et I I 2 contre I 35 p. Ioo pour les Charmoises). Ces écarts, bien que très importants, ne sont cependant pas significatifs par suite du nombre relativement faible d'animaux en comparaison.

Les Charmoises qui ont allaité 2 agneaux sont aussi fécondes que celles qui en ont allaité un seul et que les brebis taries ; par contre, dans lc troupeau de brebis croisées, les bessonnières sont légèrement moins fécondes que les mères qui allaitent un seul agneau. 
M. THÉRIEZ, G. MOLÉNAT

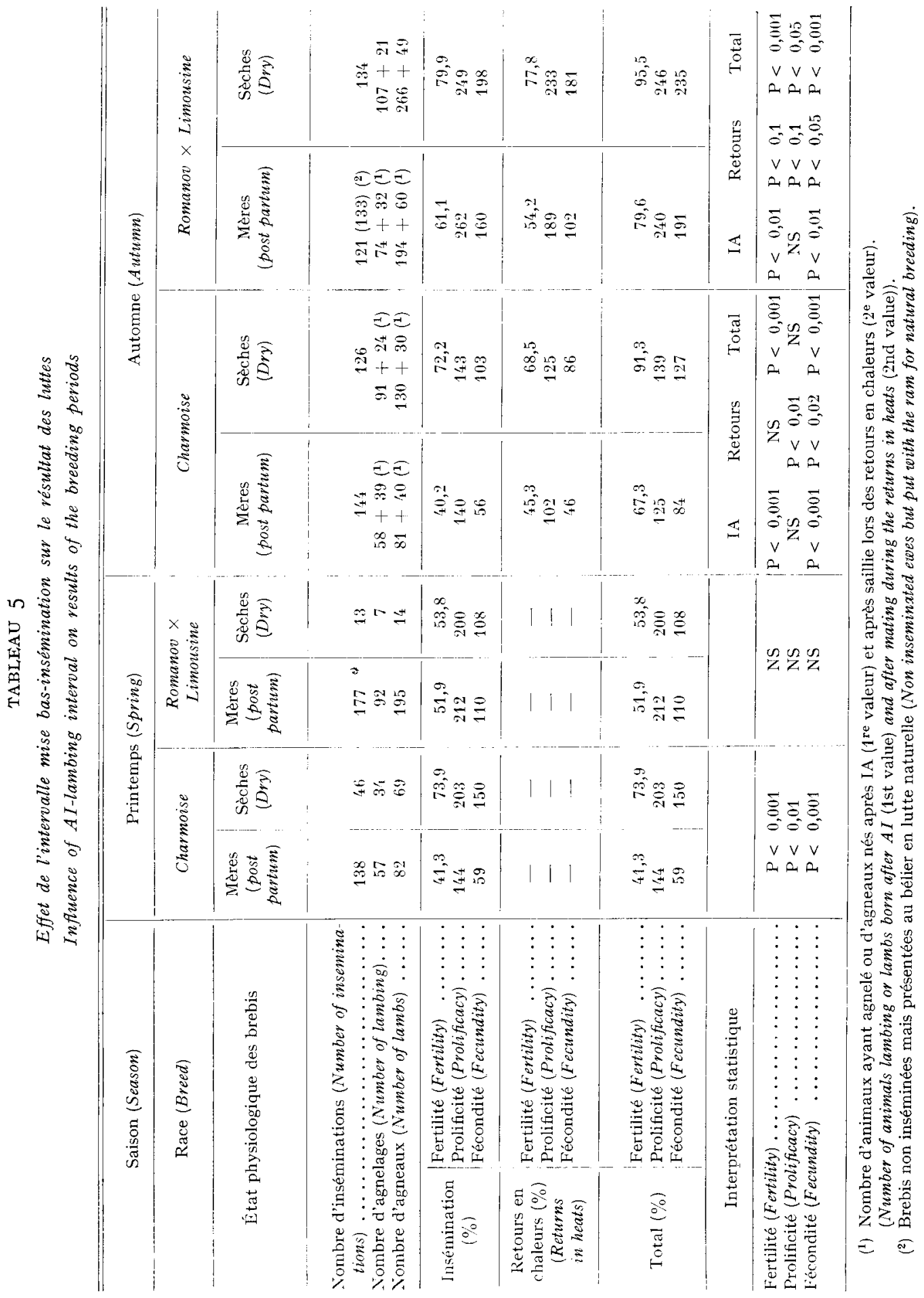




\section{TABLIAU 6}

Effets du nombre d'agneaux allaités sur la production ultérieure des brebis Effects of number of lambs suckled on subsequent production of the etes

\begin{tabular}{|c|c|c|c|c|c|c|c|c|c|c|}
\hline \multirow{3}{*}{$\begin{array}{l}\text { Période } \\
\text { de lutte } \\
\text { (Breeding } \\
\text { season) }\end{array}$} & \multirow{3}{*}{$\begin{array}{l}\text { Race des brebis } \\
\text { (Breed of er'es) }\end{array}$} & \multicolumn{9}{|c|}{ Nombre d'agneaux allaites (Number of suckling lambs) } \\
\hline & & $-\ldots$ & $\begin{array}{l}\text { Fertilité } \\
\text { (Fertility) }\end{array}$ & & & rolifi & y) & is & $\begin{array}{l}\text { 'écond } \\
\text { lectund }\end{array}$ & (c) \\
\hline & & 0 & 1 & $\ddot{2}$ & 0 & 1 & 2 & 0 & 1 & $y$ \\
\hline \multirow{2}{*}{$\begin{array}{l}\text { Printemps } \\
\text { (Spring) }\end{array}$} & Charmoises & $39,6)(6 x)$ & $42,8(56)$ & $44,4(18)$ & 148 & 137 & 162 & 58 & 59 & 72 \\
\hline & $\begin{array}{l}\text { Romanoy } \times \\
\text { Limousines }\end{array}$ & $60,2(8 x)$ & $42,8(21)$ & $46,0(68)$ & 226 & 178 & 186 & 136 & 76 & 86 \\
\hline & Charmoises & $73,5(68)$ & $59,5(12)$ & $72,7(22)$ & 122 & 136 & $11:$ & 90 & 81 & 82 \\
\hline $\begin{array}{l}\text { Automne } \\
\text { (Autumn) }\end{array}$ & $\begin{array}{l}\text { Romanoy } \times \\
\text { Limousines }\end{array}$ & $8.3,1(6.5)$ & $8=, 3(17)$ & $78,3(37)$ & 337 & $\because 71$ & 217 & 197 & 223 & 170 \\
\hline
\end{tabular}

Le nombre de brebis ayant allaité 0,1 ou \& agneaux figure entre ( ) à côté des taux de fertilité observés. (Number of ewes suckling 0,1 or 2 lambs figure betzeen ( ) near the observed fertility rates).

\section{Résultats de lutte des agnelles}

Le taux de fertilité des agnelles aprés insémination s'est élevé à 48,5 p. Ioo pour les croisées Romanov $\times$ Limousines et à 75.5 p. Ioo pour les Charmoises, lors des luttes de printemps et à 38,4 et $80 \mathrm{p}$. Ioo respectivement pour les deux races lors des luttes d'automne. Après retours en chaleurs et saillie, 76,9 et 87,5 p. Ioo des agnelles ont mis bas. Nous n'avons pas observé de différence significative en fonction de l'âge.

La prolificité des agnelles a été plus élevée après insémination de printemps qu'après insémination d'automne: 123 contre Ioo pour les Charmoises, et respectivement I 85 contre 143 et 233 contre 204 pour les croisées Romanov $\times$ Limousines âgées de 7 mois et ro mois lors de leur première lutte.

\section{Production moyenne annuelle du troupeau}

Les Charmoises sont moins fertiles que les Romanov $\times$ Limousines lorsqu'on rapporte le nombre de mises bas au nombre de luttes $(0,68$ et 0,73 ) mais un peu plus ( $\mathrm{I}, 30$ contre $\mathrm{I}, 2 \mathrm{I}$ ) lorsqu'on compare les nombres d'agnelages par femelle et par an. Cette différence est due au fait que dans le tableau 7 , nous avons inclus parmi les femelles en reproduction toutes les agnelles $R o m a-$ nov $\times$ Limousines à partir de 8 mois, âge auquel elles peuvent être saillies dans le plan de conduite de Thimonier et Mauléon (I969), alors qu'en réalité seuls 84 animaux sur un total de I83 ont été inséminés pour la première fois à cet âge.

Pendant les 4 années d'expérience, nous avons obtenu en moyenne 1,80 et 2,70 agneaux par femelle et par an pour les troupeaux de Charmoises et de Romanov $\times$ Limousines. Les valeurs pour les brebis seules, c'est-à-dire en éliminant les primipares, s'élèvent respectivement à $\mathrm{I}, 68$ et 3,09 agneaux nés/brebis/an et l'intervalle moyen entre 2 mises bas est cn moyenne de 9 mois pour les croisées, ro mois pour les Charmoises.

A l'issue de l'expérience, 242 brebis étaicnt âgées de plus de 3 ans et avaient été inséminées de 3 à 8 fois, selon leur âge. Le quart environ de ces animaux (26,4 p. I0o) a eu un taux de fertilité de I oo p. Ioo et la moitié $(52,5 \mathrm{p}$. Ioo) un taux de fertilité supérieur à $75 \mathrm{p}$. Ioo. Aucune des brebis les plus âgées, qui avaient été inséminées 7 ou 8 fois n'a été fécondée à l'issue de toutes les périodes de lutte auxquelles elle a été soumise et, seulement ro p. Ioo des brebis inséminées 5 ou 6 fois, ont eu un taux de fertilité de roo p. Ioo. 


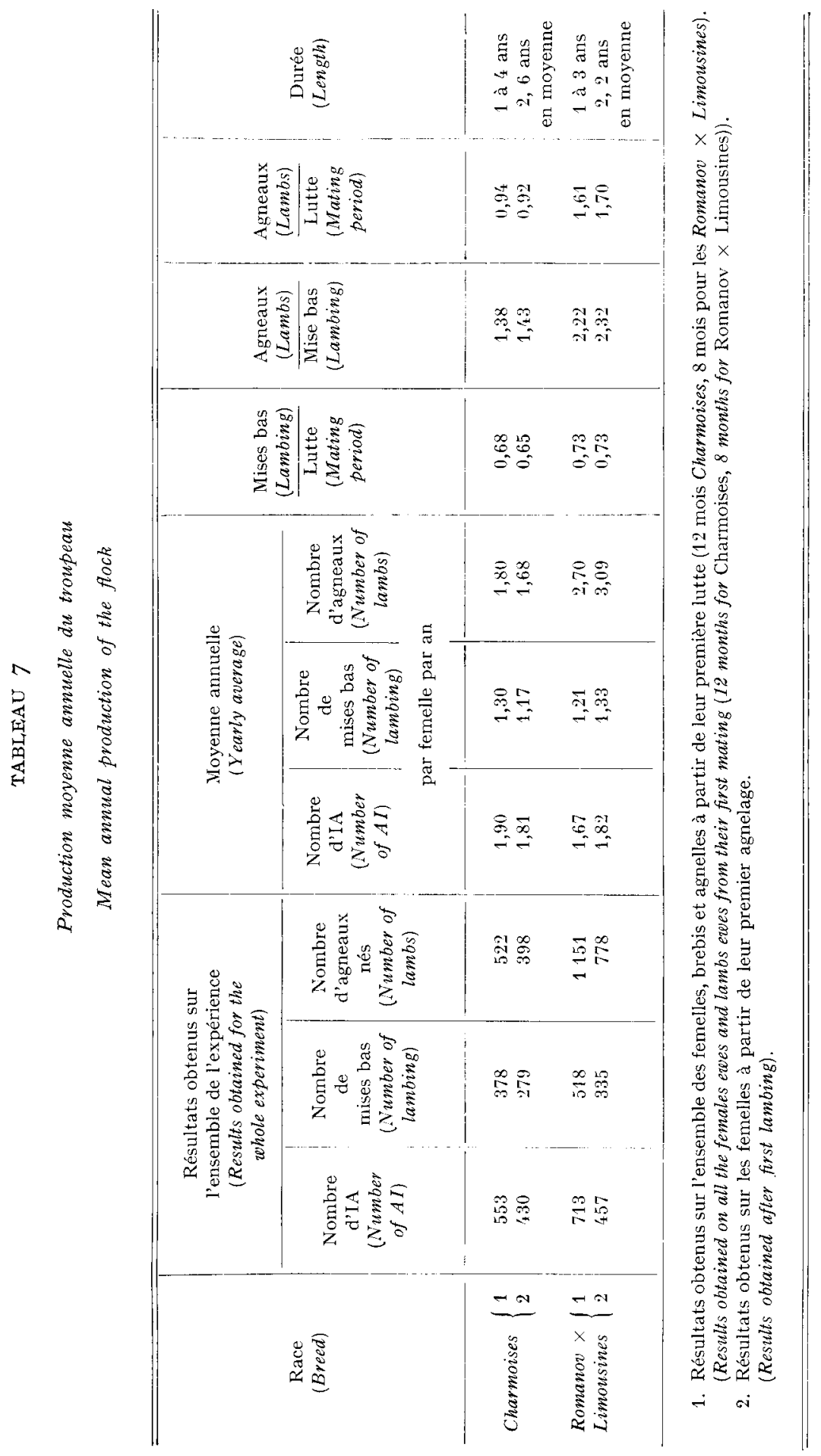




\section{DISCUSSION}

L e tarissement précoce des brebis a permis d'accroitre d'environ 8 p. Ioo la fertilité des brebis. Ce résultat est en accord avec les observations de Hunter et Lishman ( 1967 ) et de Hunter (I968). Ce dernier conclut que, s'il peut être utile de tarir les brebis pour obtenir un retour plus rapide en œstrus, il ne semble pas cependant indispensable, dans la pratique, d'avoir recours à un tel traitement.

Il y a par contre opposition entre nos résultats et ceux de MAUlíon et DaUzier (I965), Sefiddakht et al. (I97 I) ; Mallampati et al. (I97 I). Ceux-ci ont montré que, chez les brebis non soumises à un traitement hormonal, la présence de l'agneau et la lactation entraînaient une augmentation de la durée de l'anœstrus post partum et une diminution de la fertilité. Des résultats analogues ont été obtenus par LAMmING (I96I), RAHMAN et KI'T'S (I967) et ThIMONIER et al. (I968) après traitement hormonal.

Cette opposition apparente peut être due à différents facteurs et en particulier au potentiel de production laitière des brebis et à leur âge.

Barkir et Wiggins (i964), Hulet et Stormshak (I972) et Cognie et al. (1974) ont montré que les brebis qui allaitaient un seul agneau sont plus fertiles que celles qui en allaitent deux, ce qui est le cas dans le troupeau de croisées au printemps. Par contre, si nous n'avons pas observé de différence de fertilité entre brebis allaitant 0 , I ou 2 agneaux dans le troupeau de Charmoises, ce résultat peut être dû à leur faible production laitière. Leurs agneaux avaient en effet des croissances faibles, 200 à $250 \mathrm{~g} / \mathrm{j}$ pour les simples, 300 à 350 pour les portées multiples au cours des 30 premiers jours.

D'autre part, HULE'T et STORMSHAK (I972) ont observé que le sevrage précoce n'avait d'effet sur la fertilité que chez les brebis âgées de plus de 3 ans. Or nous avons réalisé notre expérience sur des animaux jeunes puisqu'en fin d'expérience les brebis les plus âgées avaient 6 ans et demi dans le troupeau de Charmoises et 4 ans dans celui de Romanoo × Limousines.

Du fait de leur fertilité plus élevée, les brebis sèches ont toujours été plus fécondes que les brebis mères, quelles que soient la race et la saison de reproduction. Ce résultat est en accord avec ceux de Thimonier et al. (I968), Thimonier et Maulíon (ig69), HULET et STORMSHAK (I972) qui constatent que le taux de fertilité augmente avec la durée de l'intervalle entre la mise bas et la saillie.

La faible fertilité après mise bas chez les brebis allaitantes provient de deux séries de faits : non involution utérine et allaitement. L'utérus ne retrouve sa texture normale qu'un certain temps après l'agnelage; il est possible que cette involution ne se produise pas à la même vitesse dans toutes les races, mais ceci reste à vérifier. Lorsque la femelle allaite, son équilibre endocrinien est modifié et ceci pourrait jouer sur la qualité des ovules ou sur la survie des oufs. Ce facteur doit avoir un rôle plus important au printemps car c'est là que l'équilibre est le plus modifié.

Lorsqu'on tarit un animal, on supprime le deuxième aspect mais non le premier ; par conséquent, l'amélioration de la fertilité des brebis taries par rapport aux allaitantes ne se produit qu'après un certain temps car la non involution utérine est peutêtre le facteur le plus important. Si on allonge l'intervalle mise bas-saillie au-delà de $47-48$ jours, c'est sans doute l'allaitement qui devient le facteur le plus important, 
ce que nous n'avons pas pu mettre en évidence car l'intervalle mise bas-IA était de 50 jours environ dans nos essais.

Cependant, le mode de conduite que nous avons adopté (fig. I) intervient également lors des luttes d'automne en accentuant les différences dues à l'état physiologique des animaux. Alors que les brebis sèches peuvent être saillies par des béliers au cours des 3 périodes successives de retour en chaleurs, les brebis mères n'ont que 2,I ou o possibilités de fécondation après l'IA.

Nous avons choisi, en début d'expérience, d'utiliser les mêmes doses de PMS pour tous les animaux, quels que soient leur âge, leur race et leur stade physiologique. Il semble que, dans cet essai, la dose de 600 UI que nous avons injectée aux brebis Charmoises au printemps était trop élevée pour les brebis sèches, ceci a entraîné une prolificité de 200 p. Ioo et a donc accentué les effets du stade physiologique sur la fécondité.

Dans le troupeau soumis à l'expérience, les brebis ont agnelé en moyenne tous les 9 ou ro mois selon leur race. Compte tenu de la durée de lasgestation et de la lactation (8 semaines), leur période de repos était donc limitée à $2-3$ mois pour celles qui allaitaient. Les brebis taries disposaient au contraire de 4 à 5 mois pour reconstituer leurs réserves corporelles, période dont la durée est voisine de celle observée dans les troupeaux soumis au rythme d'un agnelage par an. La réduction de la durée de repos et de reconstitution des réserves risque donc d'entraîner une fatigue plus importante des brebis, fatigue qui pourrait se traduire, au cours de la carrière de l'animal, par une réduction de son poids vif et une réforme plus précoce. Or nous n'avons pas observé un tel effet sur le troupeau utilisé puisque les brebis qui avaient déjà allaité étaient, à âge et à nombre de gestation identique plus lourdes que celles qui avaient été taries après leurs mises bas antérieures. Ce phénomène n'avait pas été signalé auparavant et nous en ignorons la cause. Il existerait donc un effet propre de la lactation sur l'utilisaton et la reconstitutıon des réserves corporelles et cet effet doit être très rapide car la différence de poids apparaît dès la deuxième mise bas.

L'adoption d'un rythme de reproduction accéléré, avec lutte tous les 6 mois, a permis d'obtenir $\mathrm{I}, 3$ à $\mathrm{I}, 4$ agnelage/brebis/an. Cependant la réduction à 50 jours de l'intervalle mise bas-saillie a entraîné une chute très importante de la fertilité. Un rythme de reproduction moins rapide (tous les 8 mois) nous aurait peut-être permis d'obtenir des résultats voisins, en évitant les luttes d'avril et mai, au cours desquelles nous n'avons observé aucun retours en chaleurs après le traitement progestatif, et en laissant toutes les brebis 34 à 40 jours au moins en présence du bélier après 1 'insémination.

En conclusion, dans les conditions de nos essais, le tarissement systématique des brebis dès la mise bas a amélioré légèrement la fertilité (environ $8 \mathrm{p}$. IOo). Cependant cette technique accroît les besoins en main d'ouvre (tarissement des mères et allaitement artificiel des agneaux) et le cout de production de l'agneau : il faut en effet lui apporter Io à $\mathrm{I} 2 \mathrm{~kg}$ d'un aliment d'allaitement en remplacement du lait de la mère qui peut être produit de manière plus économique, surtout en période de pâturage.

L'utilisation de cette technique doit donc être envisagée avec prudence et limitée aux cas où elle apporte une augmentation maximum de la fertilité. Dans notre essai, cette augmentation a été maximum pour les brebis Romanow $\times$ Limonsines les plus âgées au printemps. 


\title{
SUMMARY
}

\author{
INTENSIVE MANAGEMENT OF SHEEP. \\ FECUNDITY RATE OF THE EWES INSEMINATED EVERY 6 MONTHS \\ AS INFLUENCED BY DRYING-OFF IMMEDIATELY AFTER PAR'TURITION
}

A flock of I 2 Charmoise ewes (low prolificacy) and I 89 Romanov $\times$ Limousin ewes (high prolificacy) was subjected to intensive management with intervals of about 6 months between the lambing periods (fig. I). The animals were inseminated nearly 50 days after parturition and after hormonal treatment (progestagen-impregnated vaginal sponges and injection of 400 IU PMSG in autumn (October-November), 600 IU in spring (April-May).

At their first lambing, the ewes were distributed into 2 groups : the one nursing their lambs (lactating ewes) and the other separated from their lambs 24 hours after parturition (non lactating ewes), the lambs being reared artificially. This first lambing treatment (drying-off or nursing the lambs) was then repeated for each animal after each lambing during the experimental period (spring I969-summer r973). Fertility, prolificacy and fecundity of the animals were compared after each breeding season in spring and autumn (definitions, table $\mathrm{I}$ ).

Separation of the lambs immediately after parturition allowed to improve by about 8 p. Ioo the fertility rate of the ewes (non significant difference), but differences according to breed and season were obscrved. Non-lactating Romanov $\times$ Limousin ewes were more fertile in spring than the lactating one $(\mathrm{P}<0.05)$; their fertility rates were identical in autum (table 4$)$. Lactating Charmoise ewes exhibited the same fertility rate as the non-lactating ones in spring, and were a little less fertile in autumn $(0.05<\mathrm{P}<0$. ro).

Post partum drying-off did not affect the prolificacy of the ewes whatever the breed or season.

The fecundity rate of Romanov $\times$ Limousin ewes was improved by post partum drying-off in spring (0.05 $<\mathrm{P}<0.10)$.

During each breeding season, the dry ewes, i.e. the ewes which failed to lamb during the previous season and which had remained on rest for 6 months at least, were inseminated too. These ewes exhibited higher rates of fertility, prolificacy and fecundity than the post partum ewes (non lactating and lactating ewes) (table 5). The differences were highly significant for Charmoise ewes in spring and autumn and for the crossbred ewes in autumn.

No relationship was observed between the number of suckled lambs and fertility, prolificacy of the ewes, but the comparisons were made on a too small number of animals (table 6).

After an experimental period of 3 years, the ewes were weighed after lambing : those which had nursed their lambs after the previous lambings were significantly heavier $(\mathrm{P}<0.00 \mathrm{I})$ than those which had never nursed their lambs (table 3 ).

The intensive management schedule allowed to obtain I.2 and I.3 lambing per female and per year (ewes and first lamb ewes) for the Romanov $\times$ Limousin and Charmoise flocks, and $1 . \mathbf{I} 7$ and $\mathrm{x} .33$ lambing per adult ewe and per year, respectively for these two breeds. The number of lambs produced per female and per year represented 1.80 and 2.70 for the Charmoise and Romanov $\times$ Limousin and 1.68 and 3.09 per adult ewe and per year for these two breeds (tabl 7 ).

The origin of the differences between seasons and between breeds is discussed.

\section{RÉFÉRENCES BIBLIOGRAPHIQUES}

Barker H. B., Wiggins li. L., I964. Estrual activity in lactating ewes. J. Anim. Sci., 23, 973-980.

Cognie Y., Corni C., Mauléon P., 1974. The influences of lactation on fertility of ewes treated during post partum anoestrus with varinal sponges impregnated with fluorogestone acetate (Chronogest). Symposium "Physio.-Pathology of Reproduction and $A I$ ", Thessaloniki.

Colas G., I969. Technique nouvelle de reproduction des ovins : développements récents de l'insémination artificielle ovine. Journées de $l^{\prime} A F Z, 4 / 1-4 / 10$ Paris, décembre 1969.

Hulet C. V., Stormshak R., I972. Some factors affecting response of anoestrus ewes to hormone treatment. J. Anim. Sci., 34, IOII-IoIg.

Hunter G. L., I968. Increasing the frequency of pregnancy in sheep. I. Some factors affecting rebreeding during the post partum period. Anim. Breed. Abst., 36, 347-364. 
Hunter G. L., Lisinax A. W., 1967. Post partum ovulation and oestrus in spring lambing ewes, $J$. Reprod. Fert., 14, 473-475.

Girov R., Thériez M., Molf́nat (i., Aguer 1), ig7i. Influence de la variation de l'apport d'aliment concentré, avant et après l'œstrus induit par traitement hormonal, sur la fécondité des brebis. Ann. Zootech., 20, 32 I-330.

Lamming G. E., 1967. The use of hormones to control the fertility of farm animals. Proc. R. Soc. Med., $54,987-992$.

Mallampati R. S., Pope A. L., Casida L. E., i97i. liffect of sucking on post partum anoestrus in ewes lambing in clifferent season of the year. J. Anim. Sci., 32, 673-677.

Mauléon P., Daczier L., I965. Variation de la durée de l'anoestrus chez la brebis de race Ile de France. Ann. Biol, anim. Bioch. Biophys., 5, r32-143.

Rahman S. U., Kitss W. D., i967. Hormonal control of reproduction in lactating and non-lactating anoestrus ewes. Can. J. anim. Sci., 47, 65-69.

RoBinson T. J., I965. Use of progestagen-impreynated sponges inserted intra-vaginally or subcutaneously for the control of the cestrus cycle in the sheep. Nature, 206, 39-4I.

Sefidbakht N., Makarechian M., Girorban L., I97I. A note of the effect of early weaning of lambs on reproductive rate of autumn lambed Karakul ewes. Anim. Prod., 13, 565-567.

Thimonier J., Mauléon P., Cognie Y., Ortavant R., ig68. Déclenchement de l'cestrus et obtention de la gestation pendant l'anoestrus post partum chez la brebis à l'aide d'éponges vaginales imprégnées d'acétate de fluorogestone. Ann. Zootech., 17, 257-273.

Thimonier J., Matlfón P., i969. Technique nouvelle de reproduction des ovins. II. Plan de conduite d'un troupeau en vue de la production intensive d'agneaux de boucherie. Journées de l'AFZ, 5/I-5/13, Paris, décembre 1969.

Wagner J. F., Veenhtizen ]i. L., I968. Effect of lactation on reproductive performance in the ewe. J. anim. Sci., 27, I 198 (Abstr.). 